Note

\title{
A NOTE ON FACE COLORING ENTIRE WEIGHTINGS OF PLANE GRAPHS ${ }^{1}$
}

\author{
Stanislav Jendrol' And Peter Šugerek \\ Institute of Mathematics, Faculty of Science, \\ Pavol Jozef Šafárik University, \\ Jesenná 5, 04001 Košice, Slovakia \\ e-mail: stanislav.jendrol@upjs.sk \\ peter.sugerek@student.upjs.sk
}

\begin{abstract}
Given a weighting of all elements of a 2-connected plane graph $G=$ $(V, E, F)$, let $f(\alpha)$ denote the sum of the weights of the edges and vertices incident with the face $\alpha$ and also the weight of $\alpha$. Such an entire weighting is a proper face colouring provided that $f(\alpha) \neq f(\beta)$ for every two faces $\alpha$ and $\beta$ sharing an edge. We show that for every 2-connected plane graph there is a proper face-colouring entire weighting with weights 1 through 4 . For some families we improved 4 to 3 .
\end{abstract}

Keywords: entire weighting, plane graph, face colouring.

2010 Mathematics Subject Classification: 05C10, 05C15.

\section{REFERENCES}

[1] L. Addario-Berry, K. Dalal, C. McDiarmid, B.A. Reed and A. Thomason, Vertexcolouring edge-weightings, Combinatorica 27 (2007) 1-12. doi:10.1007/s00493-007-0041-6

[2] L. Addario-Berry, K. Dalal and B.A. Reed, Degree constrainted subgraphs, Discrete Appl. Math. 156 (2008) 1168-1174. doi:10.1016/j.dam.2007.05.059

[3] K. Appel and W. Haken, Every planar map is four-colorable, I. Discharging, Illinois J. Math. 21 (1977) 429-490.

[4] M. Bača, S. Jendrol', K.M. Kathiresan and K. Muthugurupackiam, Entire labeling of plane graph, (submitted).

\footnotetext{
${ }^{1}$ This work was supported by the Slovak Science and Technology Assistance Agency under the contract No. APVV-0023-10 and by Slovak VEGA grant No. 1/0652/12.
} 
[5] M. Bača, S. Jendrol', M. Miller and J. Ryan, On irregular total labellings, Discrete Math. 307 (2007) 1378-1388.

doi:10.1016/j.disc.2005.11.075

[6] J.A. Bondy and U.S.R. Murty, Graph Theory (Springer-Verlag, Heidelberg, 2008).

[7] A.J. Dong and G.H. Wang, Neighbor sum distinguishing colorings of some graphs, Discrete Math. Algorithms Appl. (2012) 4(4) 1250047. doi:10.1142/S1793830912500474

[8] E. Flandrin, J.F. Saclé, A. Marczyk, J. Przybyło and M. Woźniak, Neighbor sum distinguishing index, Graphs Combin. 29 (2013) 1329-1336. doi:10.1007/s00373-012-1191-x

[9] A. Frieze, R.J. Gould, M. Karoński and F. Pfender, On graph irregularity strenght, J. Graph Theory 41 (2002) 120-137. doi:10.1002/jgt.10056

[10] H. Grötzsch, Zur Theorie der discreten Gebilde. VII. Ein Dreifarbensatz für dreikreisfreie Netze auf der Kugel., Wiss. Z. Martin-Luther-Univ. Halle-Wittenberg, Math.Mat. Reihe 8 (1958/1959) 109-120.

[11] G. Chartrand, M.S. Jacobson, L. Lehel, O.R. Oellermann, S. Ruiz and F. Saba, Irregular networks, Congr. Numer. 64 (1988) 187-192.

[12] M. Kalkowski, A note on 1,2-conjecture, Electron. J. Combin. (to appear).

[13] M. Kalkowski, M. Karoński and F. Pfender, Vertex-coloring edge-weightings: towards the 1-2-3-conjecture, J. Combin. Theory (B) 100 (2010) 347-349. doi:10.1016/j.jctb.2009.06.002

[14] M. Karoński, T. Luczak and A. Thomason, Edge weights and vertex colours, J. Combin. Theory (B) 91 (2004) 151-157.

doi:10.1016/j.jctb.2003.12.001

[15] J. Przybyło and M. Woźniak, On 1,2 conjecture, Discrete Math. Theor. Comput. Sci. 12 (2010) 101-108.

[16] T. Wang and Q. Yu, On vertex-coloring 13-edge-weighting, Front. Math. China 3 (2008) $1-7$.

doi:10.1007/s11464-008-0041-x

[17] W. Wang and X. Zhu, Entire colouring of plane graphs, J. Combin. Theory (B) 101 (2011) 490-501.

doi:10.1016/j.jctb.2011.02.006

Received 13 July 2012

Revised 28 March 2013

Accepted 28 March 2013 